\title{
Keys, Money and Mobile Phone
}

\author{
Matthias Wagner \\ DOCOMO Euro-Labs
}

\begin{abstract}
Across cultures, genders and generations, the mobile phone has become one of the most essential objects in peoples everyday life. In this talk we will go through the evidence on how, next to money and keys, the mobile phone is the third-most thing to be carried around by almost everybody, nearly all the time. By looking at the demands and needs of todays mobile users, we discuss how Semantic technologies can add value to phones as well as to mobile services.

Walking through selected projects of DOCOMO Euro-Labs, we advocate that the success of future mobile services will largely depend on their ability to maximize their value in varying context. Contextual Intelligence in devices, mobile applications and service platforms will be needed to manage different mobile terminals, personalize content and services or to narrow down possibly very large sets of applicable services in a given situation. With this vision of Contextual Intelligence in mind, we are exploiting technologies from the Semantic Web for the mobile domain. For instance, to extend location-based service with knowledge and reasoning on places, people and things.
\end{abstract}

